\title{
Editorial:
}

\section{Adaptive and reconfigurable software systems and architectures}

Slim Kallel, Ismail Bouassida Rodruigez,

ReDCAD, University of Sfax, B.P. 1173, 3038 Sfax, Tunisia

Khalil Drira

LAAS-CNRS, Université de Toulouse, CNRS, Toulouse, France

\section{Introduction}

Adaptation is a key requirement for many software systems, such as cloud-based, cyberphysical and mobile systems. These systems are designed to satisfy different needs and expectations of users, in particular the need to take advantage of a service permanently. Such system should be able to adapt its structure and/or behaviour at runtime to cope with changes in their operating environment and user needs.

Several researchers have proposed prominent approaches to specify and implement adaptive systems in many fields including component-based systems, embedded systems, robotics, agent-based systems, artificial intelligence, and fault-tolerance. These approaches have rely on several methods and techniques such as models at runtime, software architecture, middle-wares, and programming paradigms to incorporate monitoring and adaptation capabilities.

This special issue received 43 submissions from an open call and extended versions of the best papers selected from the AROSA (Drira et al., 2014) (track on Adaptive and Reconfigurable Service-oriented and Component-based Applications and Architectures), held in conjunction with the 23rd IEEE WETICE 2014 Conference.

As a result of the review process, we accepted 14 papers, that address different issues related to the adaptive and reconfigurable systems and architectures including contextawareness, cloud computing, web services-based systems, publish-subscribe systems, cyber-physical systems, component-based systems, etc.

\section{Content of the special issue}

The work of Djoudi et al. (2016) addresses formal design and verification of context-aware systems. It proposes a formal model for these systems in order to facilitate their development and automate the context-aware adaptation decisions. Based on Maude, the authors define a domain-specific modelling language to specify the proposed model elements as well as, their corresponding functionalities. The main idea is to implement a management runtime environment that provides context reuse and dynamic representation of functional components interactions in terms of service invocation. The proposed approach is illustrated through an intelligent system for smart car controlling. The work of Liu et al. (2016) presents the design and implementation of SAND, a fault-tolerant distributed stream processing system for network analytics. The proposed processing system is designed to operate under high-speed network traffic. A strong consistency is given by the 
fault-tolerant scheme. SAND is used for the implementation of heavy traffic hitter detection as well as policy and charging control for cellular networks.

The work of Mohamed et al. (2016) proposes an extension for Open Cloud Computing Interface $(\mathrm{OCCl})$ to support the different aspects of autonomic computing. The proposed extension describes new resources and links that are generic kinds specialized using OCCI Mixins. The authors propose specific analysis rules and reconfiguration manager to generate reconfiguration actions based on reconfiguration strategies.

The work of Saoud et al. (2016) presents a credibility-based model for assessing web service trust. The proposed model focuses on dealing with strict end-users by using fuzzy clustering to determine an end-user's credibility. Deterministic and probabilistic trust models are introduced for assessing web service trust under uncertainty. Experiments are conducted on the movie-lens dataset to assess the effectiveness of the proposed approaches.

The work of Zernadji et al. (2016) introduces a method that supports web-services architects in analysing and integrating emerging quality requirements into an existing orchestration. Specifically, the method has been conceived in order to let evolve the original orchestration by in automatically integrating architectural patterns, such as adding and removing set of statements in the orchestration, matching with the desired quality aspects.

The work of Durán and Salaün (2016) presents a protocol for runtime reconfiguration of multi-components applications. It is mainly aimed at cloud based applications were creation and destruction virtual machines is an easy and frequent operation. The authors detail the different entities involved in their protocol. Virtual machines hosts several components that can export bindings in order to connect to other components. The authors use the example of a three tier web application to illustrate their description.

The work of Lahyani et al. (2016) proposes an analytical model for latency aware publish/subscribe systems on mobile ad hoc networks. The authors combine both proactive and reactive statistical analysis. The proactive analysis, uses Auto Regression or Auto Regressive Integrated Moving Average models. The reactive analysis uses approximating latency series with Gumbel distribution. The authors use also correlation method when failures are detected or predicted.

The work of Chan et al. (2016) discusses business process variants. Based on a graph-based formalization of business processes, a query language is introduced to determine suitably similar services from a repository to fi a gap in an existing underspecified process.

Sequencing constraints (causal and parallel) are taken into account in the matching process.

The work of Gerostathopoulos et al. (2016) presents a design method that addresses dependability and adaptability for cyber-physical systems. The authors suggest define a kind of models where CPS requirements are specified as invariants (representing the system goals). These invariants are iteratively refined and then mapped to components that ensure their satisfaction. These components are organized in architectures that can be adapted at runtime. 
The work of Khlif et al. (2016) presents a graph transformation-based approach for validation of quasi-synchronous checkpointing algorithms. They model algorithm execution as a happened-before graph or immediate dependency graph. The authors define a set of transformation rules to check that these graphs are exempt for Z-paths or Z-cycles. These can be used to enable automatic validation of checkpointing patterns in distributed systems.

The work of Gonzalez-Herrera et al. (2016) consists of offering a monitoring system built on top of a distributed environment dedicated to component based software development. In order to ensure an efficient monitoring, the authors propose to perform just-in-time monitoring that decreases the accumulated overhead of the monitoring system.

The work of Buisson et al. (2016) presents an approach for architectural reconfiguration including dynamic software updates. The approach is based on the combination of a reflective middleware as well as a proof assistant. The middleware is able to transform the current state of the system's architecture to the language of the proof assistant. The proof assistant is then used to define and proof the reconfiguration. The reconfiguration is then transformed to the language of the middleware to be executed.

The work of Camara et al. (2016) presents an experience feedback on the re-engineering of an industrial system so that it supports architecture-based self-adaptation. This system, called DCAS, is a cyber-physical system whose main functional goal is to monitor physical devices and manage data requested from these devices. The main extra-functional goal of this system is to preserve a certain level of performance and minimize the runtime cost, whenever devices have delays to answer requests, lowering thus the performance, or whenever active processors are under-used.

The work of Pradhan et al. (2016) proposes an integrated design tool suite for model-based development of component-based applications. Based on the design, a run-time system was developed to provide the information about the ability to meet the operational deadlines and required network quality-of-service, the schedulability of all the application tasks, and the feedback about the resilience of the system.

\section{Acknowledgements}

This special issue has been achieved by a number of collaborations: We would like to thank W.K. Chan, the special issues editor of Journal of Systems and Software for his support and help during the entire process of publication. The guest editors thank the referees who devoted part of their time to review and provide useful suggestions. Moreover, guest editors are most grateful to the authors for their valuable contributions and efforts to improve their papers in accordance with the suggestions and comments from reviewers and editors. Finally, thanks go also to the Elsevier editorial teams for their precious collaboration.

\section{References}

Buisson et al., 2016

J. Buisson, E. Cavalcante, F. Dagnat, E. Leroux, S. Martinez

Safe reconfiguration of coqcots and pycots components 
Future Gener. Comput. Syst (2016)

Camara et al., 2016

J. Camara, P. Correia, R. De Lemos, D. Garlan, B.S. Pedro Gomes, R. Ventura

Incorporating architecture-based self-adaptation into an adaptive industrial software system

Future Gener. Comput. Syst (2016)

Chan et al., 2016

N.N. Chan, N. Nonsung, W. Gaaloul

Service querying to support process variant development

Future Gener. Comput. Syst (2016)

Djoudi et al., 2016

B. Djoudi, C. Bouanaka, N. Zeghib

A formal framework for context- aware systems specification and verification

Future Gener. Comput. Syst (2016)

Drira et al., 2014

K. Drira, S. Kallel, I. Bouassida Rodriguez

Track report of adaptive and reconfigurable service-oriented and component-based

applications and architectures (AROSA 2014)

Proceedings of the Twenty Third IEEE International Conference on Enabling Technologies:

Infrastructure for Collaborative Enterprises (WETICE), IEEE Computer Society (2014), pp. 21-

22

View Record in Scopus

Durán and Salaün, 2016

F. Durán, G. Salaün

Robust and reliable reconfiguration of cloud applications

Future Gener. Comput. Syst (2016)

Gerostathopoulos et al., 2016

I. Gerostathopoulos, T. Bures, P. Hnetynka, J. Keznikl, M. Kit, F. Plasil, N. Plouzeau

Self-adaptation in software-intensive cyber-physical systems: from system goals to architecture configurations

Future Gener. Comput. Syst (2016)

Gonzalez-Herrera et al., 2016

I.Y. Gonzalez-Herrera, J. Bourcier, E. Daubert, W. Rudametkin, O. Barais, F. Fouquet, J. Jzquel, B. Baudry

Scapegoat: spotting abnormal resource usage in component-based reconfigurable software systems

Future Gener. Comput. Syst (2016)

Khlif et al., 2016

H. Khlif, H. Hadj Kacem, S.E. Pomares Hernandez, A. Hadj Kacem, C. Eichler, A.C. Simon 
An efficient validation approach for quasi-synchronous checkpointing oriented to distributed diagnosability

Future Gener. Comput. Syst (2016)

Lahyani et al., 2016

I. Lahyani, M. Jmaiel, C. Chassot

Analytical decisional model for latency aware publish/subscribe systems on MANET

Future Gener. Comput. Syst (2016)

Liu et al., 2016

Q. Liu, J.C. Lui, C. He, L. Pan, W. Fan, Y. Shi

Sand: a fault-tolerant streaming architecture for network traffic analytics

Future Gener. Comput. Syst (2016)

Mohamed et al., 2016

M. Mohamed, D. Belaid, S. Tata

Extending $\mathrm{OCCl}$ for autonomic management in the cloud

Future Gener. Comput. Syst (2016)

Pradhan et al., 2016

S.M. Pradhan, A. Dubey, T. Levendovszky, P.S. Kumar, W.A. Emfin-ger, D. Balasubramanian, W. Otte, G. Karsai

Achieving resilience in distributed software systems via self-reconguration

Future Gener. Comput. Syst (2016)

Saoud et al., 2016

Z. Saoud, N. Facia, Z. Maamar, D. Benslimane

A fuzzy-based credibility model to assess web services trust under uncertainty

Future Gener. Comput. Syst (2016)

Zernadji et al., 2016

T. Zernadji, C. Tibermacine, F. Cherif, A. Zouiouech

Integrating quality requirements in engineering web service orchestrations

Future Gener. Comput. Syst (2016) 